\title{
Supplementary Note to
}

\section{"On Stable and Strategy-Proof Rules in Matching Markets with Contracts"}

\author{
Daisuke Hirata* \\ Hitotsubashi University Hitotsubashi University
}

This Version: December, 2016

Discussion Paper No. 2016-14

\begin{abstract}
This note provides three additional results that are omitted from Hirata and Kasuya (2017) but were contained in an older version (Hirata and Kasuya, 2015).
\end{abstract}

Keywords: matching with contracts, stability, strategy-proofness, uniqueness, efficiency, irrelevance of rejected contracts

*Email: d.hirata@r.hit-u.ac.jp

†Email: yusuke.asia@gmail.com 
This note provides three additional results that are omitted from Hirata and Kasuya (2017) but were contained in an older version (Hirata and Kasuya, 2015). All the notation and definitions follow Hirata and Kasuya (2017). First, Theorem 1 of Hirata and Kasuya (2017), together with the results by Kominers and Sönmez (2016), immediately entails the following.

Definition 3. Given a number of slots $q_{h} \in \mathbb{N}$, a collection $\left(\succ_{h, s}\right)_{1 \leq s \leq q_{h}}$ of linear orders, called slot-specific priorities, over $\{x \in X: \mathrm{h}(x)=h\} \cup\{\varnothing\}$ induces a choice function for hospital $h$ as follows: For each $X^{\prime} \subset X, C_{h}\left(X^{\prime}\right)=\left\{x_{1}, \ldots, x_{q_{h}}\right\}-\{\varnothing\}$, where $x_{s}$ is recursively defined by letting

- $X_{s}^{\prime}=X_{s-1}^{\prime}-\left\{x \in X_{s-1}^{\prime}: \mathrm{d}(x)=\mathrm{d}\left(x_{s-1}\right)\right\}$, where $X_{0}^{\prime}$ and $x_{0}$ are defined to be $X^{\prime}$ and $\varnothing$, and,

- $x_{s}$ be the maximal element in $X_{s}^{\prime} \cup\{\varnothing\}$, with respect to $\succ_{h, s}$ for each $s=1, \ldots, q_{h}$.

Corollary 6. Suppose that every hospital $h \in H$ has a choice function $C_{h}(\cdot)$ that is induced by some slot-specific priorities. Then, the cumulative offer process induces the unique stable and strategy-proof rule.

Proof. In the case of slot-specific priorities, Kominers and Sönmez (2016) show that the rule induced by the cumulative offer process is both stable and strategy-proof. The uniqueness follows from our Theorem 1.

Second, along with Theorem 5 of Hatfield and Kojima (2010), Theorem 2 of Hirata and Kasuya (2017) leads to the following corollary.

Definition 4. Hospital $h$ 's choice function $C_{h}(\cdot)$ satisfies the unilateral substitutes condition if there do not exist contracts $x, y \in X$ and a subset $X^{\prime} \subset X$ of contracts such that 
(i) $\mathrm{d}(x) \notin\left\{\mathrm{d}\left(x^{\prime}\right)\right\}_{x^{\prime} \in X^{\prime}}$, (ii) $x \notin C_{h}\left(X^{\prime} \cup\{x\}\right)$, and (iii) $x \in C_{h}\left(X^{\prime} \cup\{x, y\}\right){ }^{1}$

Corollary 7. Suppose that every hospital $h \in H$ has a choice function $C_{h}(\cdot)$ satisfying the unilateral substitutes condition and the IRC condition. Then, if a stable and strategy-proof rule exists, it is induced by the doctor-proposing deferred acceptance algorithm. ${ }^{2}$

Proof. Under the supposition, a doctor-optimal stable allocation always exists and is induced by the doctor-proposing deferred acceptance algorithm (Hatfield and Kojima, 2010, Theorem 5). ${ }^{3}$ Hence, the claim immediately follows from our Theorem 2.

Lastly, we present a variant of Theorem 3 of Hirata and Kasuya (2017), which establish the second-best optimality of the doctor-optimal stable rule no matter whether it is strategy-proof or not, given its existence.

Theorem 8. Suppose that every hospital $h \in H$ has a choice function $C_{h}(\cdot)$ satisfying the IRC condition. Then, no individually rational and strategy-proof rule strictly dominates the doctoroptimal stable rule (whether strategy-proof or not).

Proof. Towards a contradiction, suppose that $f(\cdot)$ is individually rational and strategyproof, the doctor-optimal stable rule $X^{*}(\cdot)$ is well-defined, and that $f(\cdot)$ strictly dominates $X^{*}(\cdot)$. As in the proofs of Theorems $1-3$, let $\succ_{D}^{*} \in \mathscr{P}$ be a preference profile such that $f\left(\succ_{D}^{*}\right) \neq X^{*}\left(\succ_{D}^{*}\right)$ and

$$
\left[f\left(\succ_{D}\right) \neq X^{*}\left(\succ_{D}\right) \Longrightarrow \sum_{d \in D}\left|\operatorname{Ac}\left(\succ_{d}\right)\right| \geq \sum_{d \in D}\left|\operatorname{Ac}\left(\succ_{d}^{*}\right)\right|\right] \text { for all } \succ_{D} \in \mathscr{P}_{D} .
$$

Then, for the same reasoning as in the proof of Theorem 3, it follows from the stability of $X^{*}(\cdot)$ that there exists $d^{*} \in D$ with $\mathrm{x}\left(d^{*}, f\left(\succ_{D}^{*}\right)\right) \succ_{d^{*}}^{*} \mathrm{x}\left(d^{*}, X^{*}\left(\succ_{D}^{*}\right)\right) \succ_{d^{*}}^{*} \varnothing$.

\footnotetext{
${ }^{1}$ For other variants of the substitutes conditions and their relationships, see also Hatfield and Kojima $(2008,2010)$ and Afacan and Turhan (2015).

${ }^{2}$ Under the same conditions, an independent paper by Afacan (2016) characterizes the deferred acceptance as the unique stable rule that cannot be manipulated by "truncations," although it may not be fully strategy-proof.

${ }^{3}$ See also Aygün and Sönmez (2012, Theorem 5).
} 
Now, let $\succ_{D}^{* *}=\left(\succ_{d^{*}}^{* *} \succ_{D-\left\{d^{*}\right\}}^{*}\right)$, where $\succ_{d^{*}}^{* *}$ is a truncation of $\succ_{d^{*}}^{*}$ abovex $\left(d^{*}, X^{*}\left(\succ_{D}^{*}\right)\right)$, i.e., a preference such that

$$
\operatorname{Ac}\left(\succ_{d^{*}}^{* *}\right)=\left\{x \in X: x \succ_{d^{*}}^{*} \mathrm{x}\left(d^{*}, X^{*}\left(\succ_{D}^{*}\right)\right)\right\}
$$

and

$$
\left[x \succ_{d_{*}}^{* *} y \Longleftrightarrow x \succ_{d_{*}}^{* *} y\right] \text { for all } x, y \in \operatorname{Ac}\left(\succ_{d^{*}}^{* *}\right)
$$

On the one hand, the strategy-proofness of $f(\cdot)$ implies

$$
\mathrm{x}\left(d^{*}, f\left(\succ_{D}^{* *}\right)\right)=\mathrm{x}\left(d^{*}, f\left(\succ_{D}^{*}\right)\right) \neq \varnothing .
$$

On the other hand, $\mathrm{x}\left(d^{*}, X^{*}\left(\succ_{D}^{* *}\right)\right)=\varnothing$ must also hold by doctor-optimality. ${ }^{4}$ These together imply $f\left(\succ_{D}^{* *}\right) \neq X^{*}\left(\succ_{D}^{* *}\right)$, which contradicts the definition of $\succ_{D}^{*}$, and the proof is complete.

\section{References}

AfaCAN, M. O. (2016): "Characterizations of the Cumulative Offer Process," Social Choice and Welfare, $47,531-542$.

Afacan, M. O. And B. Turhan (2015): “On Relationships between Substitutes Conditions," Economics Letters, 126, 10-12.

AYGÜN, O. AND T. SÖNMEZ (2012): “The Importance of Irrelevance of Rejected Contracts in Matching under Weakened Substitutes Conditions," mimeo.

Hatfield, J. W. AND F. Kojima (2008): “Matching with Contracts: Comment," American Economic Review, 98, 1189-1194.

\footnotetext{
${ }^{4}$ If $\mathrm{x}\left(d^{*}, X^{*}\left(\succ_{D}^{* *}\right)\right) \neq \varnothing$, by construction, any $\left(h, X^{\prime \prime}\right)$ that blocks $X^{*}\left(\succ_{D}^{* *}\right)$ at $\succ_{D}^{*}$ must also block $X^{*}\left(\succ_{D}^{* *}\right)$ at $\succ_{D}^{* *}$. Thus $X^{*}\left(\succ_{D}^{* *}\right)$ is stable at $\succ_{D^{*}}^{*}$, but this contradicts the doctor-optimality of $X^{*}\left(\succ_{D}^{*}\right)$ for $\mathrm{x}\left(d^{*}, X^{*}\left(\succ_{D}^{* *}\right)\right) \succ_{d^{*}} \mathrm{x}\left(d^{*}, X^{*}\left(\succ_{D}^{*}\right)\right)$.
} 
(2010): "Substitutes and Stability for Matching with Contracts," Journal of Economic Theory, 145, 1704-1723.

Hirata, D. AND Y. KASUYA (2015): “On Stable and Strategy-Proof Rules in Matching Markets with Contracts," Working Paper No. 2015-10, Graduate School of Economics, Hitotsubashi University.

(2017): "On Stable and Strategy-Proof Rules in Matching Markets with Contracts," Journal of Economic Theory, forthcoming, doi:10.1016/j.jet.2016.12.005.

Kominers, S. D. And T. SÖnmez (2016): "Matching with Slot-Specific Priorities: Theory," Theoretical Economics, 11, 683-710. 